\title{
Threshold resummation in the rapidity distribution for a colorless particle production at the LHC
}

\section{Pulak Banerjee}

The Institute of Mathematical Sciences, Taramani, Chennai 600113, India

Homi Bhabha National Institute, Training School Complex, Anushakti Nagar, Mumbai 400085, India

E-mail: bpulakeimsc.res.in

\section{Goutam Das*}

Theory Group, Deutsches Elektronen-Synchrotron (DESY),

Notkestrasse 85, D-22607 Hamburg, Germany

E-mail: goutam.das@desy.de

\section{Prasanna K. Dhani}

The Institute of Mathematical Sciences, Taramani, Chennai 600113, India

Homi Bhabha National Institute, Training School Complex, Anushakti Nagar, Mumbai 400085,

India

E-mail: prasannakdeimsc.res.in

\section{Ravindran}

The Institute of Mathematical Sciences, Taramani, Chennai 600113, India

Homi Bhabha National Institute, Training School Complex, Anushakti Nagar, Mumbai 400085, India

E-mail: ravindra@imsc.res.in

\begin{abstract}
We present a formalism to resum large threshold logarithms to all orders in perturbative QCD for the rapidity distribution of any colorless particle at the hadron colliders. Using the derived resummed coefficients in two dimensional Mellin space, we present the rapidity distributions for the Higgs as well as for the Drell-Yan production to NNLO+NNLL accuracy at the LHC. The resummed distributions give stable prediction against the variation of unphysical renormalisation and factorisation scales in both the cases. Perturbative convergence is also improved with the inclusion of the resummed result.
\end{abstract}

Loops and Legs in Quantum Field Theory (LL2018)

29 April 2018 - 04 May 2018

St. Goar, Germany

Preprint: DESY 18-115

${ }^{*}$ Speaker. 


\section{Introduction}

Resummation of large logarithms for the rapidity distribution has been an interesting topic over the years and several results are already available to a very good accuracy for different processes. The fixed order (FO) predictions are often not reliable in certain regions of phase space where large logarithms of some kinematic variables appear. For example, at the partonic threshold, where the initial partons have just enough energy to produce the final state such as a Higgs boson or $Z / W^{ \pm}$boson or a pair of leptons in addition to soft gluons, the phase-space available for the gluons becomes severely constrained which results in large logarithms. In a truncated FO calculation, these large logarithms give unreliable results and need to be systematically resummed to all orders in perturbation theory for reliable predictions.

When talking about resummation of rapidity, two distinct approaches can be observed in QCD. One we call Catani \& Trentadue approach (or Mellin-Mellin (M-M) approach) [1] which was proposed for the $x_{F}$ distribution but can easily be extended to the rapidity distribution. In this approach the threshold limit is taken using both partonic scaling variables $z_{1}, z_{2}$ simultaneously going to the threshold limit 1 . This basically resums all the delta $\left(\delta\left(1-z_{i}\right)\right)$ terms and the distributions $\left(\left[\frac{\ln ^{n}\left(1-z_{i}\right)}{1-z_{i}}\right]_{+}\right)$arising in $z_{1}$ and $z_{2}$. Using this approach lepton pair resummation has been performed at NLL accuracy [2]. The other approach, we call Laenen \& Sterman approach (or Mellin-Fourier (M-F) approach) [3]. Here the partonic cross-section is written in terms of the scaling variable $z$ and partonic rapidity $y_{p}$ and finally the threshold limit is taken only for $z \rightarrow 1$ which resums the delta $(\delta(1-z))$ terms and the distributions $\left(\left[\frac{\ln ^{n}(1-z)}{1-z}\right]_{+}\right)$in $z$. However, for partonic $y_{p}$ only the delta $\left(\delta\left(y_{p}\right)\right)$ piece is taken. Using this approach, resummation has been performed for $W^{ \pm}$production [4] as well as Drell-Yan (DY) rapidity upto NNLL accuracy [5, 6].

We follow the M-M approach and derive an all order resummed result in two dimensional Mellin space for the rapidity distribution of any colourless state $F$ that can be produced in hadron colliders. We present our results in terms of the Mellin variables $N_{1}$ and $N_{2}$ corresponding to $z_{1}$ and $z_{2}$ respectively. In Mellin space, the limits $z_{i} \rightarrow 1$ translate into $N_{i} \rightarrow \infty$ and large logarithms proportional to $\ln \left(N_{i}\right)$ are resummed to all orders in perturbation theory. We present numerical results for resummed rapidity distributions for Higgs [7] and DY [8] productions at the LHC.

\section{Theoretical Framework}

The rapidity distribution of a colorless state $F$ at the hadron colliders can be written as

$$
\frac{d \sigma^{I}}{d y}=\sigma_{\mathrm{B}}^{I}\left(x_{1}^{0}, x_{2}^{0}, q^{2}\right) \sum_{a b=q, \bar{q}, g} \int_{x_{1}^{0}}^{1} \frac{d z_{1}}{z_{1}} \int_{x_{2}^{0}}^{1} \frac{d z_{2}}{z_{2}} \times \mathscr{H}_{a b}^{I}\left(\frac{x_{1}^{0}}{z_{1}}, \frac{x_{2}^{0}}{z_{2}}\right) \Delta_{d, a b}^{I}\left(z_{1}, z_{2}, q^{2}\right) .
$$

For brevity, the renormalization scale $\left(\mu_{R}\right)$ and the factorisation scale $\left(\mu_{F}\right)$ dependences are kept implicit in the above equation. Here the hadron level rapidity is $y=\frac{1}{2} \ln \left(p_{2} . q / p_{1} . q\right)=\frac{1}{2} \ln \left(x_{1}^{0} / x_{2}^{0}\right)$; $\tau=q^{2} / S=x_{1}^{0} x_{2}^{0}, q$ being the momentum of the final state $F, S=\left(p_{1}+p_{2}\right)^{2}$, where $p_{i}$ are the momenta of incoming hadrons $P_{i}(i=1,2)$. For the DY process, the state $F$ is a pair of leptons with invariant mass $q^{2}(I=q)$ and $\sigma^{I}=d \sigma^{q}\left(\tau, q^{2}, y\right) / d q^{2}$ whereas for the Higgs boson production through gluon (bottom anti-bottom) fusion, $I=g(b)$ and $\sigma^{I}=\sigma^{g(b)}\left(\tau, q^{2}, y\right)$. The luminosity $\mathscr{H}_{a b}^{I}$ in Eq. (2.1) is given by the product of parton distribution functions (PDFs) $f_{a}^{P_{1}}\left(x_{1}, \mu_{F}^{2}\right)$ and 
$f_{b}^{P_{2}}\left(x_{2}, \mu_{F}^{2}\right)$, renormalized at $\mu_{F}$. The partonic coefficient functions denoted by $\Delta_{d, a b}^{I}$ depend on the parton level scaling variables $z_{i},(i=1,2)$. Using factorization properties of the cross sections and renormalization group invariance, the threshold enhanced contribution to the $\Delta_{d, a b}^{I}$ denoted by $\Delta_{d, I}^{\mathrm{SV}}$ was shown to exponentiate [9] as

$$
\Delta_{d, I}^{\mathrm{SV}}=\left.\mathscr{C} \exp \left(\Psi_{d}^{I}\left(q^{2}, \mu_{R}^{2}, \mu_{F}^{2}, \bar{z}_{1}, \bar{z}_{2}, \varepsilon\right)\right)\right|_{\varepsilon=0},
$$

where the exponent $\Psi_{d}^{I}$ is both ultraviolet and infrared finite to all orders in perturbation theory. It contains finite distributions computed in $4+\varepsilon$ space-time dimensions, expressed in terms of two shifted scaling variables $\bar{z}_{1}=1-z_{1}$ and $\bar{z}_{2}=1-z_{2}$ and takes the following form:

$$
\begin{aligned}
\Psi_{d}^{I} & =\left(\ln \left(Z^{I}\left(\hat{a}_{s}, \mu_{R}^{2}, \mu^{2}, \varepsilon\right)\right)^{2}+\ln \left|\hat{F}^{I}\left(\hat{a}_{s}, Q^{2}, \mu^{2}, \varepsilon\right)\right|^{2}\right) \delta\left(\bar{z}_{1}\right) \delta\left(\bar{z}_{2}\right) \\
& -\mathscr{C}\left(\ln \Gamma_{I I}\left(\hat{a}_{s}, \mu^{2}, \mu_{F}^{2}, \bar{z}_{1}, \varepsilon\right) \delta\left(\bar{z}_{2}\right)+\left(\bar{z}_{1} \leftrightarrow \bar{z}_{2}\right)\right)+2 \Phi_{d}^{I}\left(\hat{a}_{s}, q^{2}, \mu^{2}, \bar{z}_{1}, \bar{z}_{2}, \varepsilon\right) .
\end{aligned}
$$

We have defined, $Q^{2}=-q^{2}$ and the scale $\mu$ is introduced to define the dimensionless strong coupling constant $\hat{a}_{s}=\hat{g}_{s}^{2} / 16 \pi^{2}$ in dimensional regularization, which is related to renormalised $a_{s}$ through the renormalization constant $Z\left(a_{s}\left(\mu_{R}^{2}\right)\right)$ i.e., $\hat{a}_{s}=\left(\mu / \mu_{R}\right)^{\varepsilon} Z\left(\mu_{R}^{2}\right) S_{\varepsilon}^{-1} a_{s}\left(\mu_{R}^{2}\right)$ with $S_{\varepsilon}=$ $\exp \left[\left(\gamma_{E}-\ln 4 \pi\right) \varepsilon / 2\right], \gamma_{E}=0.57721566 \cdots$ the Euler-Mascheroni constant. The definition of the double Mellin convolution $\mathscr{C}$ is given in [9] and it is understood that the regular functions resulting from various convolutions are dropped. The overall operator renormalization constant $Z^{I}$ renormalizes the bare form factor $\hat{F}^{I}$; the corresponding anomalous dimension is denoted by $\gamma_{I}$. The diagonal mass factorization kernels $\Gamma_{I I}$ remove the initial state collinear singularities. We have factored out the form factor and the mass factorization kernels in $\Delta_{d, a b}^{I}$ in such a way that the remaining soft distribution function $\Phi_{d}^{I}$ contains only soft gluon contributions. Both the form factor $\hat{F}^{I}$ and the soft distribution function $\Phi_{d}^{I}$ satisfy Sudakov type differential equations (see $[10,11]$ ) which are straightforward to solve in powers of strong coupling constant and they can be found in $[9,10,11,12]$. In terms of these solutions we arrive at the following expression (setting $\mu_{R}^{2}=\mu_{F}^{2}$ ):

$$
\begin{aligned}
\Psi_{d}^{I} & =\delta\left(\bar{z}_{2}\right)\left(\frac{1}{\bar{z}_{1}}\left\{\int_{\mu_{F}^{2}}^{q^{2} \bar{z}_{1}} \frac{d \lambda^{2}}{\lambda^{2}} A_{I}\left(a_{s}\left(\lambda^{2}\right)\right)+D_{d}^{I}\left(a_{s}\left(q^{2} \bar{z}_{1}\right)\right)\right\}\right)_{+}+\frac{1}{2}\left(\frac { 1 } { \overline { z } _ { 1 } \overline { z } _ { 2 } } \left\{A^{I}\left(a_{s}\left(z_{12}\right)\right)\right.\right. \\
& \left.\left.+\frac{d D_{d}^{I}\left(a_{s}\left(z_{12}\right)\right)}{d \ln z_{12}}\right\}\right)_{+}+\frac{1}{2} \delta\left(\bar{z}_{1}\right) \delta\left(\bar{z}_{2}\right) \ln \left(g_{d, 0}^{I}\left(a_{s}\left(\mu_{F}^{2}\right)\right)\right)+\left(\bar{z}_{1} \leftrightarrow \bar{z}_{2}\right) .
\end{aligned}
$$

Here $z_{12}=q^{2} \bar{z}_{1} \bar{z}_{2}$ and $A^{I}$ are the cusp anomalous dimensions which are known upto four loops [13]. The finite function $D_{d}^{I}$ can be expanded order by order in strong coupling and can be found from the inclusive counterpart with the use of the following identity [9, 14]:

$$
\int_{0}^{1} d x_{1}^{0} \int_{0}^{1} d x_{2}^{0}\left(x_{1}^{0} x_{2}^{0}\right)^{N-1} \frac{d \sigma^{I}}{d y}=\int_{0}^{1} d \tau \tau^{N-1} \sigma^{I}
$$

where $\sigma^{I}$ is the inclusive cross section. Comparing against $D^{I}$ from the inclusive cross section, we obtain

$$
\begin{aligned}
D_{d, 1}^{I}= & D_{1}^{I} ; D_{d, 2}^{I}=D_{2}^{I}-\zeta_{2} \beta_{0} A_{1}^{I} ; D_{d, 3}^{I}=D_{3}^{I}+\zeta_{2}\left(-\beta_{1} A_{1}^{I}-2 \beta_{0} A_{2}^{I}-2 \beta_{0}^{2} f_{1}^{I}\right)-4 \zeta_{3} \beta_{0}^{2} A_{1}^{I} ; \\
D_{d, 4}^{I}= & D_{4}^{I}+\zeta_{2}\left(-2 \beta_{1} A_{2}^{I}-\beta_{2} A_{1}^{I}-\beta_{0}\left(3 A_{3}^{I}+5 \beta_{1} f_{1}^{I}\right)-6 \beta_{0}^{2} f_{2}^{I}-12 \beta_{0}^{3} \overline{\mathscr{G}}_{1}^{I, 1}\right)-\frac{57}{5} \zeta_{2}^{2} \beta_{0}^{3} A_{1}^{I} \\
& -\beta_{0} \zeta_{3}\left(12 \beta_{0} A_{2}^{I}+10 \beta_{1} A_{1}^{I}+12 \beta_{0}^{2} f_{1}^{I}\right) .
\end{aligned}
$$


After taking double Mellin moments [15] of Eq. (2.2), we arrive at the $N_{1}-N_{2}$ space cross-section:

$$
\tilde{\Delta}_{d, I}^{(r e s)}\left(N_{1}, N_{2}\right) \equiv \tilde{\Delta}_{d, I}^{S V}(\omega)=\int_{0}^{1} d x_{1}^{0}\left(x_{1}^{0}\right)^{N_{1}-1} \int_{0}^{1} d x_{2}^{0}\left(x_{2}^{0}\right)^{N_{2}-1} \Delta_{d, I}^{S V} \equiv g_{d, 0}^{I}\left(a_{s}\right) \exp \left(g_{d}^{I}\left(a_{s}, \omega\right)\right),(2.7
$$

where $\omega=a_{s} \beta_{0} \ln \left(\bar{N}_{1} \bar{N}_{2}\right)$ (with $\bar{N}_{i}=e^{\gamma_{E}} N_{i}, i=1,2$ ). Eq. (2.7) is organised in such a way that $g_{d}^{I}\left(a_{s}, \omega\right)$ contains only $N_{1}, N_{2}$ dependent terms whereas $g_{d, 0}^{I}\left(a_{s}\right)$ are $N_{1}, N_{2}$ independent. The $N_{i}$ independent coefficients $g_{d, 0}^{I}\left(a_{s}\right)$ can be expanded in powers of $a_{s}$ as $\ln \left(g_{d, 0}^{I}\right)=\sum_{i=0}^{\infty} a_{s}^{i} l_{g_{0}}^{I,(i)}$. The exponent $g_{d}^{I}\left(a_{s}, \omega\right)$ takes the canonical form:

$$
g_{d}^{I}\left(a_{s}, \omega\right)=g_{d, 1}^{I}(\omega) \ln \left(\bar{N}_{1} \bar{N}_{2}\right)+\sum_{i=0}^{\infty} a_{s}^{i} g_{d, i+2}^{I}(\omega)
$$

To perform resummation at NNLO+NNLL accuracy, we need resummed coefficients upto $g_{d, 3}^{I}$ and the prefactors upto $l_{g_{0}}^{I,(2)}$ and those can be found in [7] ${ }^{1}$. Exponentiation of the coefficients $g_{d, i}^{I}$ resums the terms $a_{s} \beta_{0} \ln \left(\bar{N}_{1} \bar{N}_{2}\right)$ systematically to all orders in perturbation theory. The resummed result has to be properly matched with the fixed order result to avoid double counting of the logarithms. The matched cross-section takes the following form:

$$
\begin{aligned}
\frac{d \sigma^{I,(r e s)}}{d y}=\frac{d \sigma^{I,(F O)}}{d y}+ & \sigma_{B}^{I} \int_{c_{1}-i \infty}^{c_{1}+i \infty} \frac{d N_{1}}{2 \pi i} \int_{c_{2}-i \infty}^{c_{2}+i \infty} \frac{d N_{2}}{2 \pi i} e^{y\left(N_{2}-N_{1}\right)}(\sqrt{\tau})^{c_{I}-N_{1}-N_{2}} \tilde{f}_{I}\left(N_{1}\right) \tilde{f}_{I}\left(N_{2}\right) \\
& \times\left[\tilde{\Delta}_{d, I}^{(r e s)}\left(N_{1}, N_{2}\right)-\left.\tilde{\Delta}_{d, I}^{(r e s)}\left(N_{1}, N_{2}\right)\right|_{\mathrm{tr}}\right] .
\end{aligned}
$$

Here $c_{I}=-4$ for $I=g$ and 2 for $I=q$. The subscript $\operatorname{tr}$ refers to the result obtained from Eq. (2.7) by truncating at a desired accuracy in $a_{s}$. Note that the coefficients $g_{d, 0}^{I}$ and $g_{d, i}^{I}$ are functions of cusp $\left(A_{i}^{I}\right)$, collinear $\left(B_{i}^{I}\right)$, soft $\left(f_{i}^{I}\right), \mathrm{UV}\left(\gamma_{i}^{g}\right)$ anomalous dimensions, universal soft terms $G_{d, j}^{I, i}$ and process dependent constants $G_{j}^{I, i}$ of virtual corrections. These constants are known to sufficient order to perform resummation to NNLL accuracy. The $N_{i}$ dependent terms inside the square bracket appropriately multiplied with $N_{i}$ dependent PDFs, namely $\tilde{f}_{I}\left(N_{i}\right)$, have to undergo two Mellin inversions to obtain the final result in terms of $\tau$ and $y$. We have used minimal prescription advocated in [16] to perform the Mellin inversion to finally get resummed rapidity distribution.

\section{Results}

\subsection{Higgs rapidity distribution}

To perform the numerical analysis for the Higgs rapidity distribution, we have adopted the following choices of parameters: $\sqrt{S}=13 \mathrm{TeV}, M_{H}=125 \mathrm{GeV}, n_{f}=5, M_{t}=173 \mathrm{GeV}$ and used the MMHT2014 [17] PDF set with the corresponding value of strong coupling constant at each order in perturbation theory. The FO results up to NNLO are obtained using the publicly available code FEHIP [18] whereas the resummed contributions are included up to NNLL using an in-house Fortran code. To assess the scale uncertainty resulting from unphysical renormalisation and factorisation scales, we vary them between $\left[M_{H} / 2,2 M_{H}\right]$ around the central scale $\mu_{R}=\mu_{F}=$

\footnotetext{
${ }^{1}$ The $g_{d, 4}^{I}$ and $l^{I,(3)}$ coefficients can also be found in the first arXiv version of [7].
} 


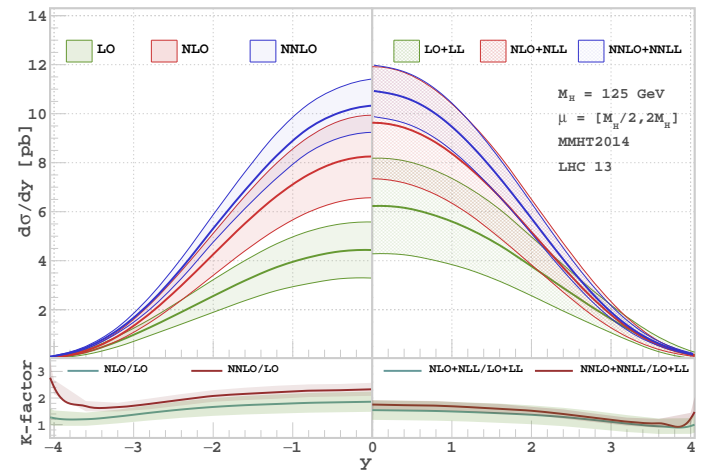

Figure 1: Higgs rapidity distributions for fixed order (left panel) upto NNLO and resummed (right panel) contributions upto NNLO+NNLL are presented with scale variation around central scale choice $M_{H}$. The respective K-factors are shown at the bottom panel.

$M_{H}$ with the constraint $1 / 2 \leq \mu_{R} / \mu_{F} \leq 2$. In Fig. (1), we have plotted the production cross section for the Higgs boson as a function of its rapidity $y$ up to NNLO in the left panel and to NNLO+NNLL in the right panel along with respective $K$-factors. We observe that the extent of overlap between consecutive orders in the resummed case is better compared to the fixed order indicating the fact that inclusion of the higher order corrections has improved the convergence of the perturbation series (see Fig. (1)). In particular, NNLO+NNLL increases approximately by $13 \%$ with respect to NLO+NLL whereas the corresponding number for NNLO over NLO is approximately $25 \%$. We also found that the choice of different central scales has minimum effect on the resummed result at NNLO+NNLL level (see Fig. (2a)). The scale uncertainties coming from the variation of $\mu_{F}$ and $\mu_{R}$ are also reduced by the inclusion of resummed contributions (Fig. (2b)).

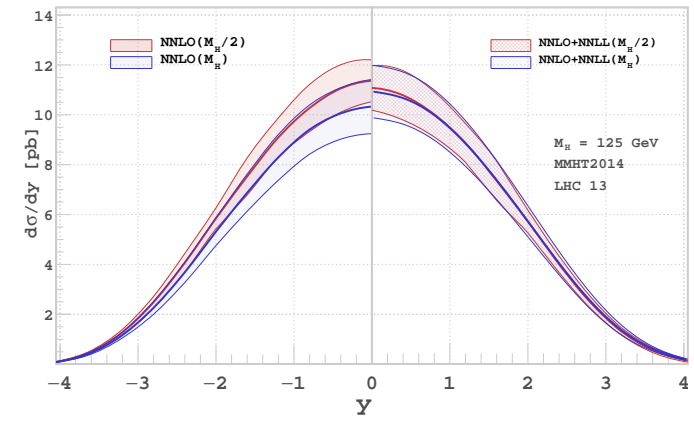

(a)

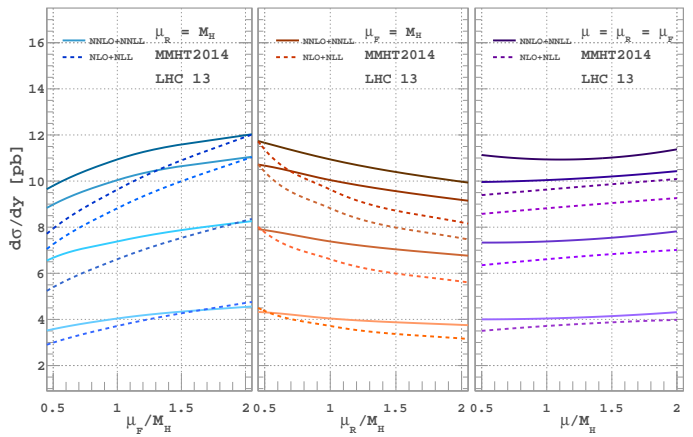

(b)

Figure 2: (a) Higgs rapidity distributions for fixed order and resummed contributions are presented with scale variation around central scale choices $M_{H} / 2$ and $M_{H}$ at NNLO+NNLL. (b) $\mu_{F}, \mu_{R}$ scale variations for different benchmark $y$ values (starting from the top $y=0,0.8,1.6,2.4$ ). 


\begin{tabular}{|l||l|l|l||l|l|l||l|l|l|}
\hline$\left(\frac{\mu_{R}}{M_{Z}}, \frac{\mu_{F}}{M_{Z}}\right)$ & LO & $\mathrm{LL}_{\mathrm{M}-\mathrm{F}}$ & $\mathrm{LL}_{\mathrm{M}-\mathrm{M}}$ & $\mathrm{NLO}$ & $\mathrm{NLL}_{\mathrm{M}-\mathrm{F}}$ & NLL $_{\mathrm{M}-\mathrm{M}}$ & $\mathrm{NNLO}$ & $\mathrm{NNLL}_{\mathrm{M}-\mathrm{F}}$ & NNLL $_{\mathrm{M}-\mathrm{M}}$ \\
\hline \hline$(2,2)$ & 72.626 & +0.988 & +3.219 & 73.450 & +1.639 & +1.796 & 70.894 & +0.630 & +0.646 \\
\hline$(2,1)$ & 63.197 & +0.768 & +2.595 & 70.625 & +0.761 & +1.017 & 70.360 & +0.292 & +0.317 \\
\hline$(1,2)$ & 72.626 & +1.095 & +3.577 & 73.535 & +1.912 & +1.760 & 70.509 & +0.510 & +0.395 \\
\hline$(1,1)$ & 63.197 & +0.851 & +2.887 & 71.395 & +0.858 & +0.901 & 70.537 & +0.248 & +0.167 \\
\hline$(1,0.5)$ & 53.241 & +0.621 & +2.216 & 67.581 & +0.156 & +0.140 & 69.834 & -0.001 & -0.094 \\
\hline$(0.5,1)$ & 63.197 & +0.953 & +3.278 & 72.355 & +0.945 & +0.681 & 70.266 & +0.091 & -0.015 \\
\hline$(0.5,0.5)$ & 53.241 & +0.695 & +2.504 & 69.259 & +0.102 & -0.154 & 70.283 & -0.039 & -0.146 \\
\hline
\end{tabular}

Table 1: Comparison of resummed results between the M-F and the M-M approaches in the minimal prescription scheme at $y=0$ for various choices of scales.

\subsection{Drell-Yan rapidity distribution}

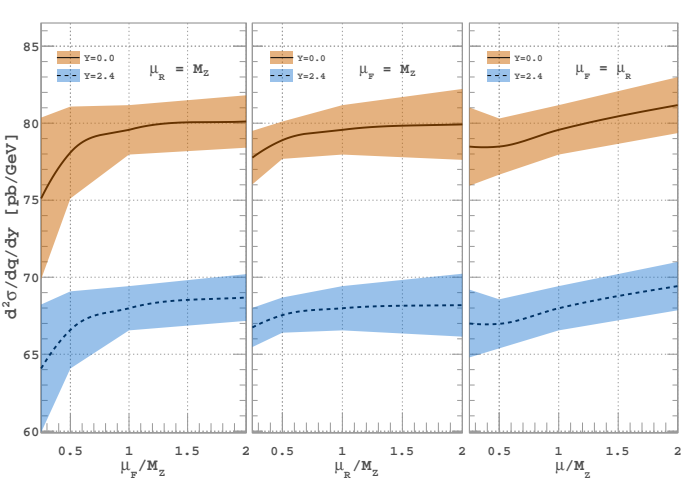

(a)

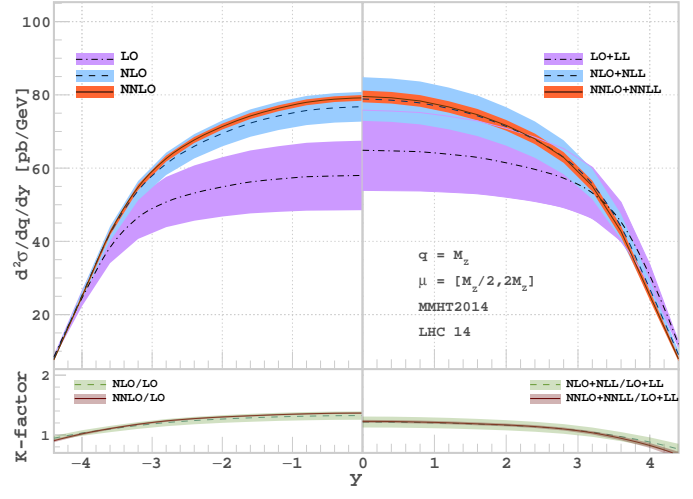

(b)

Figure 3: (a) DY cross sections against $\mu_{F}$ (left), $\mu_{R}$ (middle) and $\mu$ (right) variations at NNLO+NNLL for $14 \mathrm{TeV}$ LHC. (b) The rapidity distribution for $14 \mathrm{TeV}$ LHC at $q=M_{Z}$ with bottom panels representing the $\mathrm{K}$-factors.

For the DY rapidity distribution we choose to work at $14 \mathrm{TeV}$ LHC and focus mainly on the $Z$-peak region. The NNLO contributions are obtained from Vrap-0.9 [20]. We have performed a detailed analysis on the choice of the central scale and have found out the best prediction for the FO case is $\left(\mu_{r}, \mu_{f}\right)=(1,1) M_{Z}$ whereas in the resummed case it is $\left(\mu_{r}, \mu_{f}\right)=(1 / 2,1) M_{Z}$ (see Fig. (3a)). In the DY case, we see a better perturbative convergence for the resummed case as compared to the FO. The scale uncertainty, however, is more in the resummed case compared to the FO (Fig. (3b)). The reduced scale uncertainty at the FO is due to the large cancellation of the contributions from different partonic channels which could be accidental and might not hold at higher orders. Resummation only takes care of the large logarithms coming from the distribution in the $q \bar{q}$ channel; therefore considering only the $q \bar{q}$ channel, we get less scale uncertainty compared to the FO as expected. We have also estimated PDF uncertainties using different groups of PDFs besides 


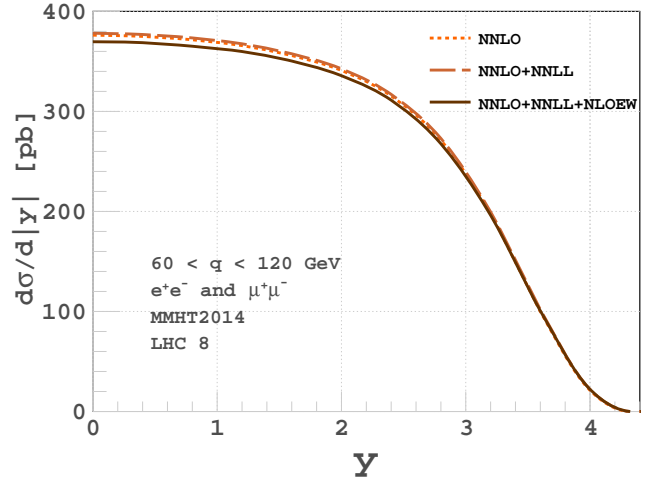

(a)

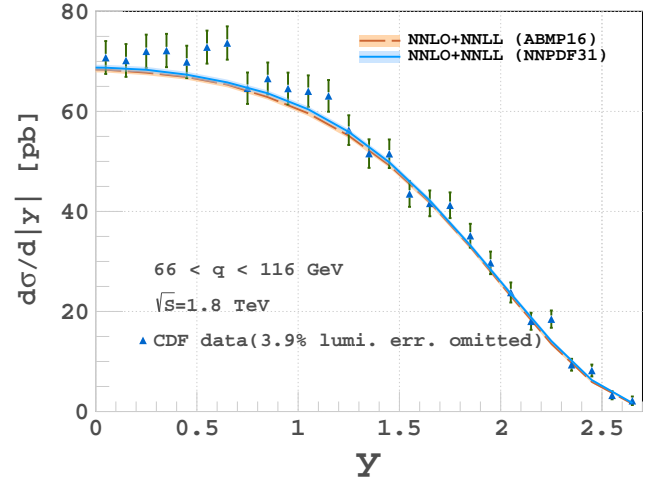

(b)

Figure 4: (a) DY rapidity distributions at NNLO+NNLL for $8 \mathrm{TeV}$ LHC in the invariant mass range $60<q<120 \mathrm{GeV}$. (b) Comparison between the resummed results and the CDF data [19] at $\sqrt{s}=1.8 \mathrm{TeV}$ in the invariant mass range $66<q<116 \mathrm{GeV}$ for two different PDF sets.

the MMHT set and we found that PDF uncertainties are consistent among different PDF groups and remain within $2 \%$ at NNLO+NNLL. We have also made a numerical comparison between the M-F and the M-M approaches keeping parameters same for both cases as in [6]. We have found a significant difference at LO+LL level; though at higher orders the differences are negligible at the level of cross-section. The M-M approach however provides a better perturbative convergence ( see Table-1). Finally we stress that at this accuracy the electro-weak (EW) corrections are important. Using the publicly available code Horace [21] we have included the EW corrections at NLO accuracy with $q$-integrated NNLO+NNLL QCD results at $8 \mathrm{TeV}$ LHC (Fig. (4a)). Moreover, we compare our prediction with the CDF data [19] for $\sqrt{S}=1.8 \mathrm{TeV}$ integrated over $q$ in the range $66<q<116 \mathrm{GeV}$ and find a very good agreement (Fig. (4b)).

\section{Conclusion}

We have developed a formalism to resum the threshold logarithms in double Mellin space for the rapidity distribution of a colorless final state $F$ produced at hadron colliders. An analytic expression of the resummed coefficients up to $\mathrm{N}^{3} \mathrm{LL}$ has been presented in terms of the double Mellin variables $N_{1}$ and $N_{2}$. As an application we have studied the role of the resummed threshold logarithms in the rapidity distribution for Higgs and the DY production at the LHC. We have performed a detailed study on the scale variations and central scale choice as well as estimated uncertainty coming from PDFs. The numerical impact of our resummation in double Mellin space has significant differences at the leading logarithmic accuracy compared to the existing results in the literature; however, we found agreement at NNLO+NNLL level. Our resummed coefficients can be used for the rapidity distribution of any colorless final state produced at the LHC. The numerical analysis presented here would be useful to understand the properties of the Higgs boson as well as it will be very useful for precise determination of PDFs at the LHC. 


\section{References}

[1] S. Catani and L. Trentadue, Resummation of the QCD Perturbative Series for Hard Processes, Nucl. Phys. B327 (1989) 323-352.

[2] D. Westmark and J. F. Owens, Enhanced threshold resummation formalism for lepton pair production and its effects in the determination of parton distribution functions, Phys. Rev. D95 (2017) 056024, [1701.06716].

[3] E. Laenen and G. F. Sterman, Resummation for Drell-Yan differential distributions, in The Fermilab Meeting DPF 92. Proceedings, 7th Meeting of the American Physical Society, Division of Particles and Fields, Batavia, USA, November 10-14, 1992. Vol. 1, 2, pp. 987-989, 1992.

[4] A. Mukherjee and W. Vogelsang, Threshold resummation for W-boson production at RHIC, Phys. Rev. D73 (2006) 074005, [hep-ph/ 0601162$].$

[5] P. Bolzoni, Threshold resummation of Drell-Yan rapidity distributions, Phys. Lett. B643 (2006) 325-330, [hep-ph/0609073].

[6] M. Bonvini, S. Forte and G. Ridolfi, Soft gluon resummation of Drell-Yan rapidity distributions: Theory and phenomenology, Nucl. Phys. B847 (2011) 93-159, [1009.5691].

[7] P. Banerjee, G. Das, P. K. Dhani and V. Ravindran, Threshold resummation of the rapidity distribution for Higgs production at NNLO+NNLL, Phys. Rev. D97 (2018) 054024, [1708 . 05706 ].

[8] P. Banerjee, G. Das, P. K. Dhani and V. Ravindran, Threshold resummation of the rapidity distribution for Drell-Yan production at NNLO+NNLL, 1805.01186.

[9] V. Ravindran, J. Smith and W. L. van Neerven, QCD threshold corrections to di-lepton and Higgs rapidity distributions beyond $N^{2}$ LO, Nucl. Phys. B767 (2007) 100-129, [hep-ph/ 0608308 ].

[10] V. Ravindran, On Sudakov and soft resummations in QCD, Nucl. Phys. B746 (2006) 58-76, [hep-ph/0512249].

[11] V. Ravindran, Higher-order threshold effects to inclusive processes in QCD, Nucl. Phys. B752 (2006) 173-196, [hep-ph/0603041].

[12] T. Ahmed, M. K. Mandal, N. Rana and V. Ravindran, Rapidity Distributions in Drell-Yan and Higgs Productions at Threshold to Third Order in QCD, Phys. Rev. Lett. 113 (2014) 212003, [1 404.6504 ].

[13] S. Moch, B. Ruijl, T. Ueda, J. A. M. Vermaseren and A. Vogt, On quartic colour factors in splitting functions and the gluon cusp anomalous dimension, 1805.09638.

[14] V. Ravindran and J. Smith, Threshold corrections to rapidity distributions of $Z$ and $W^{ \pm}$bosons beyond $N^{2}$ LO at hadron colliders, Phys. Rev. D76 (2007) 114004, [0 708 . 1689 ].

[15] S. Catani, D. de Florian, M. Grazzini and P. Nason, Soft gluon resummation for Higgs boson production at hadron colliders, JHEP 07 (2003) 028, [hep-ph / 0306211 ].

[16] S. Catani, M. L. Mangano, P. Nason and L. Trentadue, The Resummation of soft gluons in hadronic collisions, Nucl. Phys. B478 (1996) 273-310, [hep-ph/9604351].

[17] L. A. Harland-Lang, A. D. Martin, P. Motylinski and R. S. Thorne, Parton distributions in the LHC era: MMHT 2014 PDFs, Eur. Phys. J. C75 (2015) 204, [1412. 3989 ].

[18] C. Anastasiou, K. Melnikov and F. Petriello, Fully differential Higgs boson production and the di-photon signal through next-to-next-to-leading order, Nucl. Phys. B724 (2005) 197-246, [hep-ph/0501130]. 
[19] CDF collaboration, T. Affolder et al., Measurement of $d(\sigma) /$ dy for high mass Drell-Yan $e^{+} e^{-}$pairs from p $\bar{p}$ collisions at $\sqrt{s}=1.8 \mathrm{TeV}$, Phys. Rev. D63 (2001) 011101, [hep-ex/0 006025$]$.

[20] C. Anastasiou, L. J. Dixon, K. Melnikov and F. Petriello, High precision QCD at hadron colliders: Electroweak gauge boson rapidity distributions at NNLO, Phys. Rev. D69 (2004) 094008, [hep-ph/0312266].

[21] C. M. Carloni Calame, G. Montagna, O. Nicrosini and A. Vicini, Precision electroweak calculation of the production of a high transverse-momentum lepton pair at hadron colliders, JHEP 10 (2007) 109, [0710.1722]. 